Grund möchte ich auch ein paar Worte zur finanziellen Familienunterstützung in Spanien verlieren. Während eine Frau, die seit zwei Jahren in Spanien Geld verdient und Steuern zahlt, noch bis zum 1. Januar 2011 ein im Sozialversicherungsgesetz (dank der Regierung Zapateros seit 2007) geregeltes einmaliges Geldgeschenk von EUR 2.500 für die Geburt sowie monatlich EUR 100 für die ersten drei Lebensjahres des Kindes erhält, ist für Familien keine weitere finanzielle Unterstützung vorgesehen. Das Wort Rabenmutter gibt es hier dementsprechend nicht, wenn eine Mutter nach den 16 Wochen Mutterschutz nach der Geburt sofort wieder arbeiten geht. Für Krippenplätze ist gesorgt, wer Geld hat, kann sich auch eine südamerikanische oder osteuropäische Tagesmutter in der eigenen Wohnung leisten. Vor der Geburt gibt es keinen Mutterschutz, wobei die Gynäkologen mit ihren Attesten nicht geizen und somit auch die spanische Schwangere de facto vor der Geburt ihren Schutz hat. Durch strenge Gesetze ist die berufstätige Schwangere bzw. Mutter auch relativ gut geschützt, wobei mit (viel) Geld ein jeder Arbeitgeber die unliebsamen (!) Schwangeren und Mütter loswerden kann. Mein Gynäkologe hat mir bestätigt, dass viele seiner in der Privatwirtschaft arbeitenden
Patientinnen während der Schwangerschaft einem erhöhten Druck seitens ihrer Arbeitgeber ausgesetzt sind und somit erhebliche psychische Belastungen aushalten müssen. Wie lange sich das ein Land wie Spanien noch erlauben kann, soll dahin gestellt bleiben - trotz offiziell proklamierten Geburtenrückgangs sehe ich jeden Tag viele Schwangere bzw. kleine Kinder.

Kontakt zur spanischen Vereinigung von weiblichen Juristinnen „Themis“ habe ich auch schon gesucht, aber leider noch nicht aufbauen können, da sich bisher keine Frau auf meine E-Mails gemeldet hat. An Kontakten zu anderen Juristinnen gibt es bisher für mich einen von mir und einer ehemaligen Kollegin gegründeten virtuellen Stammtisch im Internetnetzwerk XING, in welchem sich nationale und internationale Juristinnen aus und in Madrid austauschen können.

Zusammenfassend kann ich aus voller Überzeugung schreiben, dass sich für mich meine Station in Madrid in jeder Hinsicht gelohnt hat. Rückblickend kann ich also jede Studentin und Referendarin nur dazu ermutigen, ins Ausland zu gehen, um dort eine Station zu absolvieren, denn „Quien no se arriesga, no gana“ („Wer nicht wagt, der nicht gewinnt”).

\title{
Und täglich ruft der Muezzin - meine Wahlstation in Kairo
}

\section{Andrea Kirsch}

Wissenschaftliche Mitarbeiterin am Lehrstuhl für Staats- und Verwaltungsrecht mit besonderer Berücksichtigung des Sozialrechts von Prof. Dr. Stefan Huster an der Ruhr-Universität Bochum

\section{Die Auswahl der Station}

Ursprünglich wollte ich meine Wahlstation bei der deutschen Botschaft in Kairo verbringen, weil ich dachte, dass dies meinem Interesse am Öffentlichen Recht näher käme. Für den Zeitraum, in dem die Station lag (11/2008 bis 1/2009), nahm die Botschaft jedoch keine Referendare an, weswegen ich mich bei der Deutsch-Arabischen Industrie- und Handelskammer bewarb. Bereits wenige Wochen nach meiner Bewerbung hatte ich die Zusage meiner späteren Ausbilderin.

Die Monate bis zur Abreise nutzte ich, um mich intensiv auf Kairo vorzubereiten, indem ich Erfahrungsberichte las, mich in Online-Foren austauschte und Reiseführer wälzte. An dieser Stelle sei allen Interessierten insbesondere der E-MailVerteiler cairoscholars (https://utlists.utexas.edu/sympa/info/ cairoscholars) empfohlen. Dort bekommt man alle nützlichen Informationen, die man für den Alltag in Kairo braucht, von Unterkunftsmöglichkeiten über Sprachlehrer und Ärzte bis hin zu Ausflugstipps.

\section{Die Station}

\section{Die Arbeit}

Die deutsch-arabische Industrie- und Handelskammer ist eine Außenhandelskammer (AHK) und vertritt als offizielle Vertretung der Deutschen Wirtschaft deren Interessen gegenüber der Politik und Verwaltung in Ägypten. Sie hilft z.B. bei der Suche nach geeigneten Geschäftspartnern, versucht bei (Handels-) Streitigkeiten zu vermitteln und wirbt in Ägypten für den Wirtschaftsstandort Deutschland.

Die Arbeit ist also sehr vielseitig und man kommt mit vielen Menschen in Kontakt. Die Arbeitssprache ist größtenteils englisch, manchmal deutsch. Was ich im Nachhinein gegenüber einer Station bei der Botschaft als großen Vorteil empfunden habe, ist die Tatsache, dass man an der AHK viel Kontakt zu Einheimischen hat. Sowohl die Hälfte der Belegschaft als auch die meisten Geschäftsleute, mit denen ich zu tun hatte, waren Ägypter. An den Botschaften hat man oft überwiegend Kontakt zu Deutschen.

In rechtlicher Hinsicht hatte ich insbesondere mit handelsrechtlichen Fragestellungen zu tun, u.a. mit UN-Kaufrecht, aber auch mit ägyptischem Recht habe ich mich das eine oder andere Mal - in englischer Übersetzung - beschäftigt. 


\section{Die Unterkunft}

Eine eigene Unterkunftsmöglichkeit seitens der AHK besteht nicht. Schon von Deutschland aus hatte ich aber über cairoscholars für den ersten Monat eine Unterkunft organisieren können. Ich teilte mir eine WG mit einer deutschen Praktikantin der AHK. Meine Unterkunft für Dezember und Januar bekam ich auch über cairoscholars. Diesmal wohnte ich mit einer Australierin und einer US-Amerikanerin zusammen.

Grundsätzlich sollte man sich potentielle WG-Zimmer vorher anschauen. Der Standard kann erheblich von dem gewohnten deutschen abweichen. Man sollte sich auch überlegen, ob man inmitten von „expats“, also Ausländern, wohnen möchte. Diese Viertel sind tendenziell teurer und das kulinarische und kulturelle Angebot ist auf die Ausländer ausgerichtet. Ich habe es bei meiner zweiten Unterkunft vorgezogen, in einem Einheimischenviertel zu wohnen. So wohnt man nicht in einer westlichen Parallelwelt, sondern kriegt mehr von dem wahren ägyptischen Leben mit.

\section{Kairo}

\section{Die Stadt}

Kairo ist einfach eine faszinierende Stadt. Sie vereint das alte Ägypten auf der einen mit der islamischen Kultur auf der anderen Seite. Sowohl die Pyramiden als auch unzählige alte und prachtvolle Moscheen gehören zum Stadtbild. Wenn man sich darauf einlässt, kann man jeden Tag etwas Neues entdecken. Auch wenn die Stadt in vielerlei Hinsicht modern erscheint, sollte man nie vergessen, dass die Bevölkerung tief religiös ist. Ich bin auch bei $27^{\circ} \mathrm{C}$ mit einem langärmligen Mantel unterwegs gewesen. Ein Kopftuch hatte ich jedoch nur auf, wenn dies in bestimmten Moscheen vorgeschrieben war.

Die Religion ist allgegenwärtig. Selbst meine Büronachbarin, die kein Kopftuch trug und sehr modisch gekleidet war, hat im Büro gebetet. Fünf Mal am Tag hört man - egal, wo man ist - den Ruf der unzähligen Muezzine. Nach einiger Zeit bin ich davon jedoch frühmorgens nicht mehr aufgewacht.

\section{Die Sprache}

Es ist durchaus möglich, ohne Arabischkenntnisse durch den Alltag zu kommen. Je weiter man sich jedoch von den Touristenzentren entfernt, desto mehr ist es zu empfehlen, einige Brocken Ägyptisch-Arabisch zu sprechen. Hocharabisch, wie man es meistens in den deutschen Sprachschulen lernt, taugt nur bedingt. Ich habe schon vor meinem Aufenthalt Sprachunterricht genommen und dann meine Kenntnisse vor Ort mit einer Privatlehrerin vertieft.

\section{Als Frau}

Gefährlich ist es nicht, sich als Frau allein in Kairo zu bewegen. Man sollte aber wissen, dass man als europäisch aussehende Frau bereits aus großer Entfernung auffällt und allein unterwegs eine Besonderheit darstellt. Viele, insbesondere junge, Männer können sich Sprüche, Pfiffe und dergleichen nicht verkneifen. Damit muss man umgehen können. Nach einiger Zeit hatte ich mir ein dickes Fell zugelegt und habe die Männer auf der Straße nicht mehr angeguckt, was ein wenig hilft.

Die Ägypter sind aber ansonsten ein sehr herzliches und hilfsbereites Volk. Gerade auch die älteren Männer haben mich sehr respektvoll behandelt. Arabischkenntnisse helfen einem dabei oft weiter und schaffen Vertrauen.

\section{Fazit}

Ich kann jeden, der mit dem Gedanken spielt, eine Station in Kairo zu verbringen, nur dazu ermutigen, es war einfach eine unvergessliche Zeit. Wer dazu Fragen hat, kann sich gerne an mich wenden.

\section{LL.M. oder Dr. jur. - eine Frauenfrage?}

\section{Dr. Anna Katharina Mangold, LL.M. (Cambridge) \\ Wissenschaftliche Mitarbeiterin, Freiburg i. Br.}

Oft machen sich Jurastudentinnen bereits im ersten Semester Gedanken darüber, ob sie einen „LL.M.“ machen sollen. Der LL.M. muss besonders attraktiv erscheinen, wenn selbst Erstsemesterinnen schon von ihm gehört haben und sich von ihm angezogen fühlen. Dem anglo-amerikanischen Universitätssystem entstammend verweist der „Master auf Laws“ mit dem Plural, Magistra der Rechte, auf die frühere Ausbildung sowohl im weltlichen als auch im kanonischen Recht. Inzwischen beschränkt sich das Studium längst auf das weltliche Recht. Das anglo-amerikanische System der universitären Jurist(inn)enausbildung unterscheidet sich deutlich vom deutschen mit seinem Abschlussziel „Erstes Staatsexamen“. So sind in den USA und Großbritannien zunächst lediglich Grundkenntnisse in Jura zu erwerben, die mit einem Bachelor belohnt werden (in England dem LL.B., Bachelor of Laws). Der Meistergrad ist dagegen vor allem für Studierende interessant, die eine ausgesprochen akademische Neigung haben oder auf einem bestimmten Gebiet vertiefte Kenntnisse erwerben wollen.

Für deutsche Studierende kann einer dieser beiden Gründe ausschlaggebend sein, einen LL.M. in Erwägung zu ziehen. Darüber hinaus aber sehen vor allem international tätige Kanzleien und Wirtschaftsunternehmen den Titel gerne, verspricht er doch vertiefte Sprachkenntnisse, insbesondere juristische. Zudem ist das Masterstudium mit einer Dauer von nur einem akademischen Jahr ein zeitlich überschaubares Projekt, das keinen allzu schweren Einschnitt in die übrige berufliche und persönliche Lebensplanung bedeutet. 